\title{
Paired- and induced paired-domination in (E,net)-free graphs
}

\author{
Oliver Schaudt \\ Institut für Informatik \\ Universität zu Köln \\ Weyertal 80, 50931 Cologne, Germany \\ schaudt@zpr.uni-koeln.de
}

May 11, 2011

\begin{abstract}
A dominating set of a graph is a vertex subset that any vertex belongs to or is adjacent to. Among the many well-studied variants of domination are the so-called paired-dominating sets. A paired-dominating set is a dominating set whose induced subgraph has a perfect matching. In this paper, we continue their study.

We focus on graphs that do not contain the net-graph (obtained by attaching a pendant vertex to each vertex of the triangle) or the E-graph (obtained by attaching a pendant vertex to each vertex of the path on three vertices) as induced subgraphs. This graph class is a natural generalization of (claw,net)-free graphs, which are intensively studied with respect to their nice properties concerning domination and hamiltonicity. We show that any connected (E,net)-free graph has a paired-dominating set that, roughly, contains at most half of the vertices of the graph. This bound is a significant improvement to the known general bounds.

Further, we show that any (E,net, $C_{5}$ )-free graph has an induced paireddominating set, that is a paired-dominating set that forms an induced matching, and that such set can be chosen to be a minimum paireddominating sets. We use these results to obtain a new characterization of (E,net, $C_{5}$ )-free graphs in terms of the hereditary existence of induced paired-dominating sets. Finally, we show that the induced matching formed by an induced paired-dominating set in a (E,net, $C_{5}$ )-free graph can be chosen to have at most two times the size of the smallest maximal induced matching possible.

keywords: domination, paired-domination, induced paired-domination, induced matchings, (E,net)-free graphs.
\end{abstract}

MSC: 05C69

\section{Introduction}

Let $G$ be a graph. If $S$ and $T$ are two vertex subsets such that every member of $T$ either belongs to $S$ or has a neighbor among $S$, then $S$ is said to dominate $T$. A dominating set $X$ of a graph $G$ is a vertex subset that any 
vertex of $G$ either belongs to or is adjacent to, i.e. $X$ dominates $V(G)$. There is a lot of literature dealing with the concept of dominating sets and its many variants. An introduction into the field of domination in graphs is the book by Haynes, Hedetniemi and Slater [1]. Among the common variants of domination is the concept of paired-domination. A paired-dominating set $P$ is a dominating set such that the induced subgraph, denoted $G[P]$, has a perfect matching. Note that any isolate-free graph, i.e. any graph without isolated vertices, has a paired-dominating set. The minimal size of a paired-dominating set, the paireddomination number, is denoted $\gamma_{p}(G)$. A paired-dominating set of size $\gamma_{p}(G)$ is said to be minimum. An inclusionwise minimal paired-dominating set is said to be minimal. Paired domination was introduced by Haynes and Slater [2] in 1998 with the following application in mind. The members of a paired-dominating set can be thought of as guards dominating the graph, and every guard has a partner providing backup. Paired domination received a lot of attention in the literature and is still an active topic. Among many others, some very recent papers on paired-domination were written by Dorbec and Gravier [3], Mynhardt and Schurch [4] and the author [5].

An induced matching of a graph $G$ is a subset of the edges, no two edges of which are adjacent or share a common vertex. Induced matchings were introduced by Cameron [6], and are well studied now. There are also some combinations of induced matchings and domination problems, e.g. efficient edge domination (see [7] among others) and efficient total domination (see [8] among others). Another natural combination of paired-domination and induced matchings, generalizing those concepts mentioned above, are the so-called induced paired-dominating sets. An induced paired-dominating set is a dominating set such that the induced subgraph forms an induced matching. However, it is an $\mathcal{N} \mathcal{P}$-complete problem to decide if a given graph has an induced paireddominating set [9]. If a graph $G$ has an induced paired-dominating set, the minimal size of such a set, the induced paired-domination number, is denoted $\gamma_{i p}(G)$. An induced paired-dominating set of size $\gamma_{i p}(G)$ is said to be minimum. Induced paired-dominating sets were introduced and first studied, according to our knowledge, by Haynes, Lawson and Studer [10] and later by Zelinka [11]. They are also studied (as dominating induced matchings) by Telle [9].

The complete bipartite graph $K_{1,3}$ is often called the claw. The graph $E$ is obtained from the claw by subdividing two of its three edges exactly once. The graph net is obtained by attaching a pendant vertex to any vertex of the triangle $K_{3}$. The graphs $C_{5}$, net and $E$ are displayed in Figure 1. Let $G$ and $H$ be two arbitrary graphs. $G$ is said to be $H$-free, if $H$ is not a subgraph of $G$. If $\mathcal{H}$ is a set of graphs, $G$ is $\mathcal{H}$-free if $G$ is $H$ free for all $H \in \mathcal{H}$.
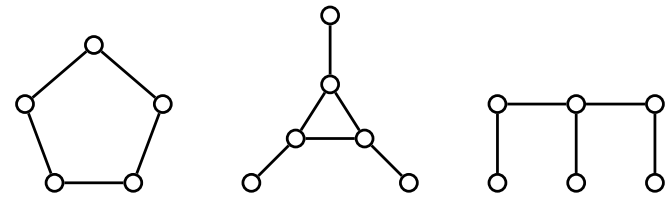

Figure 1: the graphs $C_{5}$, net and $E$.

There is some literature concerning $\{$ claw, net $\}$-free graphs, dealing with domination and hamiltonicity problems: It was shown by Damaschke [12] that 
a connected graph is $\{$ claw, net $\}$-free iff each of its connected induced subgraphs has a hamiltonian path. Later, Brandstädt and Dragan [13] studied \{claw, net $\}$ free graphs in view of their linear and circular structure. They proved that a connected \{claw, net $\}$-free graph either has a doubly dominating induced cycle or a dominating pair, i.e. a pair of vertices such that any connecting path is a dominating set. Furthermore, problems concerning hamiltonicity of $\{$ claw, net $\}$ free graphs were studied by Kelmans [14] and Brandstädt, Dragan and Köhler [15]. In this paper, we deal with the problem of paired-domination and induced paired-domination restricted to $\{E$, net $\}$-free graphs, a natural generalization of $\{$ claw, net $\}$-free graphs.

\section{The main results}

This section presents our main results. The proofs are given in section 3 .

Our first observation, arising from theorems of Tuza [16] and Bacsó [17], is the following:

Lemma 1. If $G$ is a connected $\{E$, net $\}$-free graph, then $G$ has a connected dominating set $X$ such that $G[X]$ is a path.

A bound on $\gamma_{p}$ for $\{E$, net $\}$-free graphs is obtained by the following theorem by Dorbec and Gravier [3]. There, the graph $K_{1, r}^{*}$ is obtained from $K_{1, r}$ by subdividing each edge exactly once.

Theorem 1 (Dorbec, Gravier [3]). Let $G$ be a connected graph of order $n \geq 3$. If $G$ is $K_{1, a+2}^{*}$-free for some $a \geq 1$, then

$$
\gamma_{p}(G) \leq \frac{2(a n+1)}{2 a+1}
$$

The bound is sharp.

We observe that $E$ is an induced subgraph of $K_{1,3}^{*}$. Thus if $G$ is an $E$-free connected graph of order $n \geq 3$, then $G$ is also $K_{1,3}^{*}$-free. Theorem 1 gives

$$
\gamma_{p}(G) \leq \frac{2 n+2}{3}
$$

However, (1) is not asymptotically sharp for $\{E, n e t\}$-free graphs, i.e. $2 / 3$ is not an optimal factor (see Theorem 2 ). Using Lemma 1 , we obtain a better bound, which roughly says that $\gamma_{p}$ is at most half the number of vertices of the graph considered:

Theorem 2. Let $G=(V, E)$ be a connected $\{E$, net $\}$-free graph of order $n \geq 2$. Then

$$
\gamma_{p}(G) \leq 2\left\lceil\frac{n}{4}\right\rceil
$$

The bound is sharp.

Note that $\gamma_{p}\left(P_{k}\right)=2\left\lceil\frac{k}{4}\right\rceil$ for all $k \geq 2$ as was observed by Haynes and Slater $[2]$. Hence, $(2)$ is attained by connected graphs with arbitrary large values of $\gamma_{p}(G)$. 
As the proof of Theorem 2 shows, an isolate-free connected $\{E$, net $\}$-free graph has a paired-dominating set $P$ such that $G[P]$ is the disjoint union of single edges with at most one path $P_{4}$. That is, $P$ is closed to be an induced paired-dominating set. However, there are $\{E$, net $\}$-free graphs that do not have an induced paired-dominating set (e.g. the cycle $C_{5}$ ). As the next theorem shows, if $C_{5}$ is forbidden, induced paired-dominating sets always exist and they can be chosen to be minimum paired-dominating sets:

Theorem 3. Let $G$ be an isolate-free graph that is $\left\{E\right.$, net, $\left.C_{5}\right\}$-free. Then $G$ has an induced paired-dominating set that is a minimum paired-dominating set. In particular, $\gamma_{p}(G)=\gamma_{i p}(G)$.

This leads us to the following characterization, parts of which were already proven before by the author [5].

Theorem 4. Let $G$ be a graph. The following statements are equivalent:

1. Any isolate-free induced subgraph of $G$ has an induced paired-dominating set.

2. Any isolate-free induced subgraph of $G$ has an induced paired-dominating set that is a minimum paired-dominating set.

3. $G$ is $\left\{E\right.$, net, $\left.C_{5}\right\}$-free.

Our next result bounds $\gamma_{i p}$ from above in terms of a parameter related to induced matchings. We denote by $\mathrm{im}_{-}(G)$ the minimal size of an inclusionwise maximal induced matching of $G$ (sometimes called the lower induced matching number). An inclusionwise maximal induced matching of size $i m_{-}(G)$ is called a minimum maximal induced matching. This concept was studied, among others, by Orlovich and Zverovich [18] and with Finke and Gordon [19]. Since any induced paired-dominating set forms an induced matching, each graph $G$ that has an induced paired-dominating set fulfills

$$
2 i m_{-}(G) \leq \gamma_{i p}(G)
$$

On the other hand, $\gamma_{i p}(G)$ is bounded from above by $i m_{-}(G)$ in the following way:

Theorem 5. Let $G$ be an isolate-free graph such that any isolate-free induced subgraph has an induced paired-dominating set. Then

$$
\gamma_{i p}(G) \leq 4 i m_{-}(G)
$$

That is, there is an induced paired-dominating set such that its induced matching is at most two times larger than a minimum maximal induced matching.

The bound (3) is attained by $P_{5}$. We do not know if the bound is also sharp for connected graphs with arbitrary large values of $\gamma_{i p}$.

\section{The proofs}

\subsection{Proof of Lemma 1 and Theorem 2}

Let $D$ be a class of connected graphs. $\operatorname{Dom}(D)$ is defined to be the class of connected graphs whose any connected induced subgraph $H$ has a connected 
dominating set $X$ such that $H[X]$ is isomorphic to a graph of $D$. For example, $\operatorname{Dom}\left(\left\{P_{k}: k \in \mathbb{N}\right\}\right)$ is the set of connected graphs whose any connected induced subgraph $H$ has a connected dominating set $X$ such that $H[X]$ is a path.

Tuza [16] (and independently Bacsó [17]) gives the following characterization. Note that the leaf graph $F(G)$ of a graph $G$ is obtained by attaching a pendant vertex to each of the non-cutting vertices of $G$.

Theorem 6 (Tuza [16]). Let D be a nonempty class of connected graphs closed under taking connected induced subgraphs. The minimal forbidden induced subgraphs of $\operatorname{Dom}(D)$ are the cycle $C_{t+2}$ if $P_{t} \notin D$ but $P_{t-1} \in D$ and the leaf graphs of the minimal forbidden subgraphs of $D$.

We observe that the class $\left\{P_{k}: k \in \mathbb{N}\right\}$ equals the set of connected graphs that do not contain a cycle or the claw as induced subgraph. Now we are in the position to prove Lemma 1:

Proof of Lemma 1. Let $G$ be a connected $\{E$, net $\}$-free graph. Then $G$ does not contain the leaf graph of $C_{3}$ as induced subgraph, since $F\left(C_{3}\right) \cong$ net. Further, for all $k \geq 4, E$ is an induced subgraph of $F\left(C_{k}\right)$. Hence, $G$ does not contain the leaf graph of a cycle as induced subgraph. Finally, since the leaf graph of the claw contains $E$ as induced subgraph, $G \in \operatorname{Dom}\left(\left\{P_{k}: k \in \mathbb{N}\right\}\right)$ by Theorem 6 . Thus $G$ has a connected dominating set that induces a path.

Proof of Theorem 2. Let $G=(V, E)$ be a connected $\{E$, net $\}$-free graph of order $n \geq 2$. By Lemma $1, G$ has a connected dominating set that induces a path. Among the connected dominating sets inducing a path, let $X$ be a minimal set, i.e. each of the two endvertices of the path $G[X]$ has a private neighbor with respect to $X$. If $|X|=1$, then $\gamma_{p}(G)=2$ and hence (2) holds. Thus we can assume that $|X| \geq 2$. Let $v_{1}, v_{2}, \ldots, v_{r}$ be a consecutive ordering of the vertices of $X$, i.e. $N\left(v_{1}\right) \cap X=\left\{v_{2}\right\}, N\left(v_{2}\right) \cap X=\left\{v_{1}, v_{3}\right\}$, and so on. Furthermore, let $v_{0}$ be the private neighbor of $v_{1}$ and let $v_{r+1}$ be the private neighbor of $v_{r}$.

In the following, we construct a paired-dominating set $P \subseteq\left(X \cup\left\{v_{r+1}\right\}\right)$ of $G$, say with $k$ pairs $p_{1}, p_{2}, \ldots, p_{k}$. We will ensure that $v_{r+1} \notin p_{i}$ for all $1 \leq i \leq k-1$, i.e. $v_{r+1} \notin P \backslash p_{k}$.

Further, we construct an injective function

$$
f: P \backslash p_{k} \rightarrow V \backslash\left(\left(P \backslash p_{k}\right) \cup\left\{v_{r+1}\right\}\right) .
$$

The injectivity of $f$ and $v_{r+1} \notin P \backslash p_{k}$ imply

$$
\begin{aligned}
n & \geq\left|P \backslash p_{k}\right|+\left|\left(P \backslash p_{k}\right) \cup\left\{v_{r+1}\right\}\right| \\
& \geq 2\left|P \backslash p_{k}\right|+1 \\
& \geq 4 k-3 .
\end{aligned}
$$

The fact that $\gamma_{p}(G) \leq 2 k$ gives $\gamma_{p}(G) \leq(n+3) / 2$. Note that $\gamma_{p}(G)$ is always an even number and thus it suffices to show that if $(n+3) / 2 \geq 2 i$ for some $i$, then also $2\lceil n / 4\rceil \geq 2 i$. This is clear since

$$
(n+3) / 2 \geq 2 i \Rightarrow n \geq 4 i-3 \Rightarrow\lceil n / 4\rceil \geq i \Rightarrow 2\lceil n / 4\rceil \geq 2 i .
$$

Recall the consecutive ordering $v_{1}, v_{2}, \ldots, v_{r}$ of the vertices of $X$. We iteratively construct $P$ and $f$ by the following procedure. We start with $P_{1}=p_{1}=$ 
$\left\{v_{1}, v_{2}\right\}$ and $f_{1}\left(v_{1}\right)=v_{0}, f_{1}\left(v_{2}\right)=v_{3}$. It is clear that the partial function $f_{1}$ is injective. Starting with $i=1$, we iteratively add a pair $p_{i+1}$ to the set $P_{i}$ to obtain the set $P_{i+1}$. Thereby, we define $f_{i+1}$ as an extension of $f_{i}$ to the members of $p_{i+1}$ (as long as $v_{r} \notin p_{i+1}$ ) and keep $f_{i+1}$ injective as an invariant. When the procedure terminates, we have obtained a paired-dominating set $P$ and an injective function $f$ as described above.

We initialize $P_{1}=p_{1}=\left\{v_{1}, v_{2}\right\}, f_{1}\left(v_{1}\right)=v_{0}, f_{1}\left(v_{2}\right)=v_{3}$ and $i=1$. Then we proceed with the following steps:

1. Let $j$ be such that $p_{i}=\left\{v_{j}, v_{j+1}\right\}$.

2. If $r \leq j+3$, then let $p_{i+1}=\left\{v_{r}, v_{r+1}\right\}$ and $P=P_{i} \cup p_{i+1}$. Further, let $f=f_{i}$. Terminate the procedure.

(Logic of step 2: If $r=j+3$, we do not need to consider $v_{j+2}$ as a member of $p_{i+1}$ (see proof of Claim 1). We can finish the procedure without extending $f_{i}$ to the members of $p_{i+1}$ in view of (4).)

3. If $r \geq j+4$ and not every member of $N\left(v_{j+3}\right) \backslash X$ is dominated by $P_{i}$, let $p_{i+1}=\left\{v_{j+3}, v_{j+4}\right\}$ and let $P_{i+1}=P_{i} \cup p_{i+1}$. Extend $f_{i}$ to $f_{i+1}$ by letting $f_{i+1}\left(v_{j+3}\right)$ be an arbitrary member of $N\left(v_{j+3}\right) \backslash X$ not dominated by $P_{i}$ and $f_{i+1}\left(v_{j+4}\right)=v_{j+5}$. Increment $i$ by 1 and go to step 1 .

(Logic of step 3: Since $G$ is $E$-free, we do not need to consider $v_{j+2}$ as a member of $p_{i+1}$ (see proof of Claim 1). Then $v_{j+3}$ is included in $P_{i+1}$ to guarantee that $P_{i+1}$ dominates all neighbors of the set $\left\{v_{1}, v_{2}, \ldots, v_{j+3}\right\}$ and $v_{j+4}$ is added to $P_{i+1}$ to be the matching partner of $v_{j+3}$ in $G\left[P_{i+1}\right]$. After the step, all neighbors of the set $\left\{v_{1}, v_{2}, \ldots, v_{j+4}\right\}$ are dominated by $P_{i+1}$.)

4. If $r \geq j+4$ and every member of $N\left(v_{j+3}\right) \backslash X$ is dominated by $P_{i}$, let $p_{i+1}=\left\{v_{j+4}, v_{j+5}\right\}$ and let $P_{i+1}=P_{i} \cup p_{i+1}$. If $v_{r} \in p_{i+1}$, let $f=f_{i}$, $P=P_{i+1}$ and terminate the procedure. Otherwise, extend $f_{i}$ to $f_{i+1}$ by letting $f_{i+1}\left(v_{j+4}\right)=v_{j+3}$ and $f_{i+1}\left(v_{j+5}\right)=v_{j+6}$. Increment $i$ by 1 and go to step 1 .

(Logic of step 4: Again, we do not need to consider $v_{j+2}$ as a member of $p_{i+1}$. As $v_{j+3}$ does not have a neighbor outside of $X$ that is not dominated by $P_{i}$, it is not needed in the set $P_{i+1}$. But since $v_{j+3}$ has to be dominated, we add $v_{j+4}$ to $P_{i+1}$. Then $v_{j+5}$ is added to $P_{i+1}$ to be the matching partner of $v_{j+4}$ in $G\left[P_{i+1}\right]$. After the step, all neighbors of the set $\left\{v_{1}, v_{2}, \ldots, v_{j+5}\right\}$ are dominated by $P_{i+1}$. If $v_{r}$ is among the pair $p_{i+1}$, then we can finish the procedure without extending $f_{i}$ to the members of $p_{i+1}$ in view of (4).)

Claim 1. Let $P$ and $f$ be constructed by the procedure stated above. Then $P$ is a paired-dominating set and $f$ is injective.

Proof of Claim 1. Let $P$ and $f$ be constructed by the procedure stated above and let $P$ consist of $k$ pairs $p_{1}, p_{2}, \ldots, p_{k}$. Note that $P \subseteq\left(X \cup\left\{v_{r+1}\right\}\right), p_{1}=$ $\left\{v_{1}, v_{2}\right\}$ and that $v_{r} \in p_{k}$. Furthermore, $P$ dominates every member of $X$.

To see that $P$ is a paired-dominating set of $G$, assume the contrary. Then, as $G[P]$ has a perfect matching (any member of $P$ is contained in a pair $p_{i}$ for some $i$ ), $P$ is not a dominating set (otherwise it would be a paired-dominating 
set). Since $X$ is a dominating set, there is a minimal index $3 \leq l \leq r-1$ such that there is a vertex $w \in N\left(v_{l}\right) \backslash X$ that is not dominated by $P$. Thus $v_{l} \notin P$. Since every member of $X$ is dominated by $P$, either $v_{l-1} \in P$ or $v_{l-1} \notin P$ but $v_{l-2} \in P$.

First assume that $v_{l-1} \notin P$ but $v_{l-2} \in P$. Let $v_{l-2}$ be a member of the pair $p_{i}$. Then in the construction of the pair $p_{i+1}$, step 3 would be applied to $v_{l}$, since $w \in N\left(v_{l}\right) \backslash X$ is not dominated by $P_{i}$. But then $v_{l} \in P_{i+1} \subseteq P$, a contradiction.

Thus $v_{l-1} \in P$ and so $v_{l-2} \in P$, too. If $v_{l+1} \in P$, then $v_{l+2} \in P$ and thus $G\left[\left\{v_{l-2}, v_{l-1}, v_{l}, v_{l+1}, v_{l+2}, w\right\}\right] \cong E$, a contradiction. Hence, $v_{l+1} \notin P$ and so $w \notin N\left(v_{l+1}\right)$, since otherwise step 3 would be applied to $v_{l+1}$ (as described above). Hence, $G\left[\left\{v_{l-2}, v_{l-1}, v_{l}, v_{l+1}, v_{l+2}, w\right\}\right] \cong E$ again, a contradiction.

To see that $f$ is injective, we observe that if $p_{i}=\left\{v_{j}, v_{j+1}\right\}$ is a pair of $P$ (with $i \leq k-1)$, then $f\left(v_{j}\right) \neq f\left(v_{j+1}\right)$. Furthermore, in view of the steps 3,4 and 2 , it is clear that the image of $f_{i}$ (denoted $\operatorname{im} f_{i}$ ) is dominated by the set $P_{i}$, for all $1 \leq i \leq k-1$. On the other hand, no member of $\operatorname{im} f \backslash \operatorname{im} f_{i}$ is dominated by $P_{i}$, for all $1 \leq i \leq k-1$. This means that $f$ is injective.

As described above, Claim 1 proves (2).

\subsection{Proof of Theorem 3 and Theorem 4}

Proof of Theorem 3. Let $G$ be an isolate-free graph that is $\left\{E\right.$, net, $\left.C_{5}\right\}$-free. Among the minimum paired-dominating sets of $G$ we choose the set $P$ to be minimal with respect to the number of edges in $G[P]$. Since $P$ is a paireddominating set, $G[P]$ has a perfect matching $M$. We claim that $M$ is an induced matching of $G$, i.e. $P$ is an induced paired-dominating set of $G$. If $|P|=2$, we are done. Thus we can assume that $|P| \geq 4$. Let $\{a, b\}$ and $\{c, d\}$ be two distinct edges of $M$. We claim that $G[\{a, b, c, d\}]$ contains no edges but $\{a, b\}$ and $\{c, d\}$. Since $\{a, b\}$ and $\{c, d\}$ are arbitrary, this claim completes the proof.

We assume for contradiction that $G[\{a, b, c, d\}]$ contains other edges but $\{a, b\}$ and $\{c, d\}$. For symmetry we can assume that $G[\{a, b, c, d\}]$ is identical to either one of the graphs $G_{1}, G_{2}, G_{3}, G_{4}$ or $G_{5}$ displayed in Figure 2.
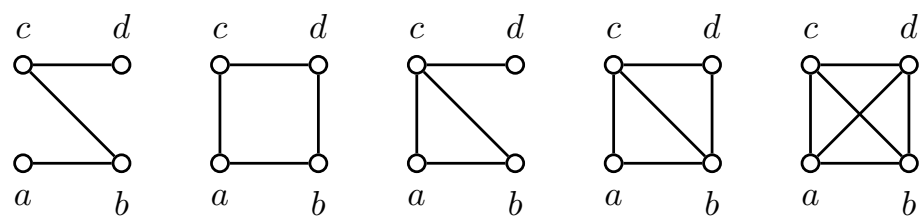

Figure 2: $G_{1}, G_{2}, G_{3}, G_{4}$ and $G_{5}$.

First we assume that $G[\{a, b, c, d\}]=G_{1}$. Since $P$ is a minimum paireddominating set, $P \backslash\{a, d\}$ is not a paired-dominating set. In fact, it is not even a dominating set, since $G[P \backslash\{a, d\}]$ still has a perfect matching. Assume for contradiction that neither $a$ nor $d$ has a private neighbor with respect to $P$. Therefore, every vertex not dominated by $P \backslash\{a, b\}$ is adjacent to both $a$ and $d$. Let $u$ be any vertex not dominated by $P \backslash\{a, b\}$. Then $G[a, b, c, d, u] \cong C_{5}$, a contradiction. Thus we can assume, without loss of generality, that $a$ has a private neighbor $u$ with respect to $P$. Let $P^{\prime}=(P \backslash\{b\}) \cup\{u\}$. Since $u$ is a private neighbor of $a, G\left[P^{\prime}\right]$ has fewer edges than $G[P]$. Hence, $P^{\prime}$ is not a 
paired-dominating set of $G . P^{\prime}$ is not even a dominating set, since $G\left[P^{\prime}\right]$ still has a perfect matching (we can substitute $\{a, b\}$ by $\{a, u\}$ in $M$ ). Hence, there is a neighbor $v$ of $b$ not dominated by $P^{\prime}$. But then $G[\{a, b, c, d, u, v\}] \cong E$, a contradiction.

Therefore, $G[\{a, b, c, d\}]$ is identical to $G_{2}, G_{3}, G_{4}$ or $G_{5}$. To shorten the proof, we make the following general observation: Since $P$ is a minimum paireddominating set, $P \backslash\{a, b\}$ is not a paired-dominating set. It is not even a dominating set, since $G[P \backslash\{a, b\}]$ still has a perfect matching. Assume for contradiction that neither $a$ nor $b$ has a private neighbor with respect to $P$. Hence, every vertex not dominated by $P \backslash\{a, b\}$ is adjacent to both $a$ and $b$. Let $u$ be any vertex not dominated by $P \backslash\{a, b\}$. Then $P^{\prime}=(P \backslash\{b\}) \cup\{u\}$ is a minimum paired-dominating set of $G$. Since $(N(u) \cap P) \backslash\{b\}=\{a\}, G\left[P^{\prime}\right]$ has fewer edges than $G[P]$, a contradiction. Thus we can assume, without loss of generality, that $a$ has a private neighbor $a^{\prime}$ with respect to $P$. By edgeminimality again, $(P \backslash\{b\}) \cup\left\{a^{\prime}\right\}$ is not a dominating set of $G$. Thus there is a vertex $b^{\prime}$ that is a private neighbor of $b$ with respect to $P$ and not adjacent to $a^{\prime}$. Again, $(P \backslash\{c, d\}) \cup\left\{a^{\prime}, b^{\prime}\right\}$ is not a dominating set. Hence, there is a vertex $w \notin P$ with $N(w) \cap P \subseteq\{c, d\}$ that is not adjacent to $a^{\prime}$ or $b^{\prime}$.

If $G[\{a, b, c, d\}]=G_{2}$, we can assume that $c$ is adjacent to $w$. But then $G\left[a, b, c, a^{\prime}, b^{\prime}, w\right] \cong E$, a contradiction.

If $G[\{a, b, c, d\}]=G_{3}$, then $G\left[a, b, c, d, a^{\prime}, b^{\prime}\right] \cong$ net, a contradiction.

If $G[\{a, b, c, d\}]=G_{4}$, we have the following two cases: If $c$ is adjacent to $w$, then $G\left[a, b, c, a^{\prime}, b^{\prime}, w\right] \cong$ net, a contradiction. If $d$ is adjacent to $w$, then $G\left[a, b, d, a^{\prime}, b^{\prime}, w\right] \cong E$, a contradiction.

Last we assume that $G[\{a, b, c, d\}]=G_{5}$. Further we can assume that $c$ is adjacent to $w$. But then $G\left[a, b, c, a^{\prime}, b^{\prime}, w\right] \cong$ net, a contradiction.

Proof of Theorem 4. Let $G$ be an isolate-free graph.

If $G$ is $\left\{E\right.$, net, $\left.C_{5}\right\}$-free, $G$ has an induced paired-dominating set that is a minimum paired-dominating set by Theorem 3. Hence, 3 implies 1 and 2 .

On the other hand, it is easy to see that none of the graphs in the set $\left\{E\right.$, net, $\left.C_{5}\right\}$ have an induced paired-dominating set. Therefore, both of the conditions 1 and 2 imply 3 . This completes the proof.

\subsection{Proof of Theorem 5}

Proof of Theorem 5. We assume for contradiction that there is a smallest number $k$ such that there is an isolate-free graph $G$ with $i m_{-}(G)=k$ that fulfills the assumption of Theorem 5 but violates (3). By Theorem $4, G$ is $\left\{E\right.$, net, $\left.C_{5}\right\}$-free.

First we assume $k=1$. Let $\{m=\{u, v\}\}$ be a minimum maximal induced matching of $G$. Let $U$ be the vertices that are not dominated by $\{u, v\}$. We assume for contradiction that $U$ is not an independent set, i.e. there is an edge $e \in E(G[U])$. By choice of $U, e$ can be added to $\{m\}$ and the resulting set would still be an induced matching of $G$, a contradiction. Hence, $U$ is an independent set. This fact will be used in the proof several times.

If $U=\emptyset,\{u, v\}$ is an induced paired-dominating set of $G$, a contradiction. Thus $|U| \geq 1$. In each of the following case distinctions we show that the set $\{u, v\}$ can be extended to a paired-dominating set of size 4, i.e. $\gamma_{p}(G) \leq 4 \mathrm{im}(G)$. But then the following contradiction appears: By Theorem $3, \gamma_{i p}(G)=\gamma_{p}(G)$ and hence (3) holds for $G$. If $|U|=1$, we choose an arbitrary neighbor of 
the unique vertex $w \in U$, say $w^{\prime}$, and observe that the set $\left\{u, v, w, w^{\prime}\right\}$ is a paired-dominating set.

If $|U|=2$, say $U=\left\{w, w^{\prime}\right\}$, we first assume that $N(w) \subseteq N\left(w^{\prime}\right)$. We choose an arbitrary neighbor $w^{\prime \prime}$ of $w$ and observe that $\left\{u, v, w, w^{\prime \prime}\right\}$ is a paireddominating set of $G$. The case $N\left(w^{\prime}\right) \subseteq N(w)$ is dealt with in a similar way. Now we assume that $N(w)$ and $N\left(w^{\prime}\right)$ are incomparable. Thus there are two distinct vertices, say $x$ and $x^{\prime}$, such that $x$ is a neighbor of $w$ and $x^{\prime}$ is a neighbor of $w^{\prime}$. In particular, $x$ and $x^{\prime}$ do not belong to $U$. We claim that $G\left[\left\{u, v, x, x^{\prime}\right\}\right]$ has a perfect matching, i.e. $\left\{u, v, x, x^{\prime}\right\}$ is a paired-dominating set. If $x$ is adjacent to $x^{\prime}$, the case is clear. Thus we assume that $x$ is not adjacent to $x^{\prime}$. If neither $x$ nor $x^{\prime}$ are adjacent to $u$, both are adjacent to $v$. But then $G\left[\left\{u, v, x, x^{\prime}, w, w^{\prime}\right\}\right] \cong E$, a contradiction. Hence, $x$ or $x^{\prime}$ is adjacent to $u$. By symmetry the same holds for $v$. Therefore, $G\left[\left\{u, v, x, x^{\prime}\right\}\right]$ has a perfect matching and thus $\left\{u, v, x, x^{\prime}\right\}$ is a paired-dominating set.

So $|U| \geq 3$. Clearly, the set $W=(N(u) \cup N(v)) \backslash\{u, v\}$ dominates $U$, i.e. every vertex of $U$ has a neighbor in $W$. We choose the set $W^{\prime} \subseteq W$ to be inclusionwise minimal with the property that every vertex of $U$ has a neighbor in $W^{\prime}$. By minimality, for every vertex $w \in W^{\prime}$ there is a vertex $w^{\prime} \in U$ with $N\left(w^{\prime}\right) \cap W^{\prime}=\{w\}$. If $\left|W^{\prime}\right|=1$, we add $W^{\prime}$ and an arbitrary member of $U$ to $\{u, v\}$ and obtain a paired-dominating set of size 4 . If $\left|W^{\prime}\right|=2$, let $x$ and $y$ be the two members of $W^{\prime}$ and let $x^{\prime}$ and $y^{\prime}$ be members of $U$ with $N\left(x^{\prime}\right) \cap W^{\prime}=\{x\}$ and $N\left(y^{\prime}\right) \cap W^{\prime}=\{y\}$. If $G[\{u, v, x, y\}]$ has a perfect matching, $\{u, v, x, y\}$ is a paired-dominating set of size 4 . If $G[\{u, v, x, y\}]$ does not have a perfect matching, it is isomorphic to $K_{1,3}$ (the only connected graph on 4 vertices without a perfect matching). But then $G\left[\left\{u, v, x, y, x^{\prime}, y^{\prime}\right\}\right] \cong E$, a contradiction. Thus $\left|W^{\prime}\right| \geq 3$. Let $x, y$ and $z$ be any three members of $W^{\prime}$ and let $x^{\prime}, y^{\prime}$ and $z^{\prime}$ be members of $U$ with $N\left(x^{\prime}\right) \cap W^{\prime}=\{x\}, N\left(y^{\prime}\right) \cap W^{\prime}=\{y\}$ and $N\left(z^{\prime}\right) \cap W^{\prime}=\{z\}$. If $G[\{x, y, z\}]$ is connected, either $G[\{x, y, z\}] \cong P_{3}$ or $G[\{x, y, z\}] \cong K_{3}$. Then $G\left[\left\{x, y, z, x^{\prime}, y^{\prime}, z^{\prime}\right\}\right] \cong E$ or $G\left[\left\{x, y, z, x^{\prime}, y^{\prime}, z^{\prime}\right\}\right] \cong$ net respectively, but both cases are contradictory. Hence, $G[\{x, y, z\}]$ is not connected.

First we assume that $x$ is adjacent to $y$ and so $z$ is isolated in $G[\{x, y, z\}]$. Furthermore, we assume that $x$ and $y$ have a common neighbor in $\{u, v\}$, say $u$. Then $z$ is not adjacent to $u$, since otherwise $G\left[\left\{u, x, y, z, x^{\prime}, y^{\prime}\right\}\right] \cong$ net, a contradiction. Hence, $z$ is adjacent to $v$. If both $x$ and $y$ are not adjacent to $v$, then $G\left[\left\{u, v, x, y, x^{\prime}, y^{\prime}\right\}\right] \cong$ net, a contradiction. On the other hand, if $x$ and $y$ are both adjacent to $v$, then $G\left[\left\{v, x, y, z, x^{\prime}, y^{\prime}\right\}\right] \cong n e t$, a contradiction. Thus we can assume that $x$ is adjacent to $v$ and $y$ is not adjacent to $v$. But then $G\left[\left\{v, x, y, z, x^{\prime}, y^{\prime}\right\}\right] \cong E$, a contradiction. Therefore, $x$ and $y$ do not have a common neighbor among $\{u, v\}$. Without loss of generality, we can assume that $z$ is adjacent to $u$. Then $G\left[\left\{u, x, y, z, x^{\prime}, y^{\prime}\right\}\right] \cong E$, a contradiction. Using a symmetric argumentation, we see that $\{x, y, z\}$ must be an independent set.

If $x, y$ and $z$ have a common neighbor in $\{u, v\}$, say $u$, then $G\left[\left\{u, x, y, z, x^{\prime}, y^{\prime}\right\}\right] \cong$ $E$, a contradiction. Hence, we can assume that $N(x) \cap\{u, v\}=\{u\}$ and $N(y) \cap\{u, v\}=\{v\}$. Further, we can assume that $z$ is adjacent to $u$. If $z$ is adjacent to $v$, then $G\left[\left\{u, v, x, y, z, z^{\prime}\right\}\right] \cong$ net, a contradiction. Thus $z$ is not adjacent to $v$. But then $G\left[\left\{u, v, x, z, x^{\prime}, z^{\prime}\right\}\right] \cong E$, a contradiction.

Since the assumption $k=1$ was shown to be contradictory, $k \geq 2$.

Let $G$ fulfill $i m_{-}(G)=k$. Let $M$ be a minimum maximal induced matching of $G$ and let $m=\{u, v\} \in M$ be arbitrary. Let $W=N(u) \cup N(v)$ and let $U$ 
be the vertices that are isolated in $G[V \backslash W]$. We observe that both the graphs $G[W \cup U]$ and $G[V \backslash(W \cup U)]$ are isolate-free. Since $\{m\}$ is a minimum maximal induced matching of $G[W \cup U]$, im $(G[W \cup U])=1$. Further, $i m_{-}(G[V \backslash(W \cup$ $U)]) \leq k-1$, since in $G$ the edge $m$ can be added to any induced matching of $G[V \backslash(W \cup U)]$. By choice of $k,(3)$ holds for $G[W \cup U]$ and $G[V \backslash(W \cup U)]$ and thus also for $G$. This completes the proof.

\section{References}

[1] T.W. Haynes, S.T. Hedetniemi, P.J. Slater, Fundamentals of Domination in Graphs, Marcel Dekker, Inc., New York, 1998.

[2] T.W. Haynes, P.J. Slater, Paired-domination in graphs, Networks 32 (1998), pp. 199-206.

[3] P. Dorbec, S. Gravier, Paired-domination in subdivided star-free graphs, Graphs and Combinatorics 26 (2010), pp. 43-49.

[4] C.M. Mynhardt, M. Schurch, Paired domination in prisms of graphs, Discussiones Mathematicae Graph Theory 31 (2011), pp. 5-23.

[5] O. Schaudt, Total domination versus paired-domination, manuscript 2011.

[6] K. Cameron, Induced matchings, Discrete Applied Mathematics 24 (1989), $97-102$.

[7] D.L. Grinstead, P.J. Slater, N.A. Sherwani, N.D. Holmes, Efficient edge domination problems in graphs, Information Processing Letters 48 (1993), pp. 221-228.

[8] O. Schaudt, On weighted efficient total domination, manuscript (2010).

[9] J.A. Telle, Complexity of domination-type problems in graphs, Nordic Journal of Computing 1 (1994), pp. 157-171.

[10] T.W. Haynes, L.M. Lawson, D.S. Studer: Induced-Paired Domination in Graphs, Ars Combinatoria 57 (2000), pp. 111-128.

[11] B. Zelinka, Induced-paired domatic numbers of graphs, Mathematica Bohemica 127 (2002), pp. 591-596.

[12] P. Damaschke, Hamiltonian-hereditary graphs, manuscript (1990).

[13] A. Brandstädt, F.F. Dragan, On linear and circular structure of (claw,net)free graphs, Discrete Applied Mathematics 129 (2003), pp. 285-303.

[14] A. Kelmans, On Hamiltonicity of \{claw,net $\}$-free graphs, Discrete Mathematics 306 (2006), pp. 2755-2761.

[15] A. Brandstädt, F.F. Dragan, E. Köhler, Linear Time Algorithms for Hamiltonian Problems on (Claw,Net)-Free Graphs, SIAM J. Comput. 30 (2000), pp. $1662-1677$.

[16] Z. Tuza, Hereditary domination in graphs: Characterization with forbidden induced subgraphs, SIAM J. Discrete Math. 22 (2008), pp. 849-853. 
[17] G. Bacsó, Complete description of forbidden subgraphs in the structural domination problem, Discrete Mathematics 309 (2009), pp. 2466-2472.

[18] Y.L. Orlovich, I.É. Zverovich, Maximal induced matchings of minimum/maximum size, manuscript (2004).

[19] G. Finke, V. Gordon, Y.L. Orlovich, I.É. Zverovich, Approximability results for the maximum and minimum maximal induced matching problems, Discrete Optimization 5 (2008), pp. 584-593. 